\title{
Arbuscular mycorrhiza fungi, NPK (I5-|5-|5) and cow dung interaction in sustainable cassava production and food security
}

\begin{abstract}
Increasing human population coupled with the depletion and degradation of soil resources constitutes a threat to food security in sub-Saharan Africa. Sequel to this, the growth, performance and yield of cassava (Manihot esculenta L.) were assessed using pure culture of arbuscular mycorrhiza fungus - Glomus deserticola, NPK (15:15:15) and Cow dung singly and in combination with each other at four treatment levels under field conditions. Control experiment was also set up without any treatment. The experiment was factorial, in completely randomized block design and replicated four times. Cassava stem cuttings, $18 \mathrm{~cm}$ length were planted in well tilled soil at a distance of $1 \mathrm{~m}$ apart and allowed to grow for six months. During the study; plant height, stem girth, leaf area, leaf chlorophyll content and yield were assessed. At harvest, fresh and dry tuber weights were measured. Data collected were subjected to one way analysis of variance and means separated using Tukey's HSD test ( $\mathrm{p} \leq 0.05)$. The study showed that Glomus deserticola in combination with NPK produced cassava plants with significant differences $(p<0.05)$ in height, stem girth, leaf area, chlorophyll content and yield. Arbuscular mycorrhiza fungus enhanced nutrient uptake of cassava plants. The balanced fertilization and amendment of the experimental soil with adequate nutrients supply provided useful agronomic information on the performance and yield of cassava.
\end{abstract}

Summary: Although some sub-Saharan African countries have for a number of year's experienced significant agricultural success, demographic growth associated with high rates of erosion and land degradation continue to have an impact on food security in this part of the world. In this study, the growth and production of cassava (Manihot esculenta L.) was assessed by the contribution of pure culture of a mycorrhizal to arbuscules (Glomus deserticola), mineral fertilizers (NPK 15-15-15), cow manure and their combinations. The test was carried out on plots of $1 \mathrm{~m} \times 1 \mathrm{~m}$. On each plot, a cut of nearly $18 \mathrm{~cm}$ length was buried in the previously plowed soil. The experimental method consisted of a complete random block with a factorial plan of four repetitions. An equivalent number of control plots have not been processed. Vegetative growth parameters, including height of the plant, circumference of the stem and leaf surface were followed for six months, then the chlorophyll content of the leaves and yield were determined. These data were subjected to a simple variance analysis and a Tukey HSD test was performed to determine the significant differences between averages $(\mathrm{p} \leq 0.05)$. The use of Glomus deserticola in combination with NPK 15-15-15 mineral fertilizers has resulted in significant vegetative growth and yield significantly higher than other treatments. This suggests that this fungus improves the absorption of nutrients in cassava.

Keywords: soil fertilization, amendment, manihot esculenta, growth performance, food security
Volume 7 Issue 4 - 2017

\author{
Chukwuka KS,' Okechukwu RU,'2 Umukoro \\ BO,' Obiakara $\mathrm{MC}^{\prime}$ \\ 'Department of Botany, University of Ibadan, Nigeria \\ ${ }^{2}$ Cassava Transformation Agenda Project, International Institute \\ of Tropical Agriculture, Nigeria
}

Correspondence: Chukwuka KS, Department of Botany,

University of Ibadan, Ibadan-Nigeria,

Email kanayodrchukwuka97@gmail.com

Received: July 24, 2017| Published: August 22, 2017

\section{Introduction}

Cassava (Manihot esculenta Crantz) is a woody shrub that is widely cultivated in many tropical and subtropical regions of the world. It is propagated from stem cuttings and produces edible energy-rich tubers. Dry cassava tubers contain up to $90 \%$ carbohydrates, of which starch is dominant Montagnac et al. ${ }^{1}$ The importance of this crop in food security is reflected in its remarkably increasing production over the last decade. In 2014, about 270 million tonnes constituting about $54 \%$ of global production were produced in Africa FAOSTAT. ${ }^{2}$ This crop is reputed for its remarkable tolerance to abiotic stresses due to its biochemical and physiological adaptations, intermediate $\mathrm{C}_{3}-\mathrm{C}_{4}$ photosynthetic pathway, reduction in leaf area index coupled with a relatively high stomatal sensitivity in response to water shortages and unaltered photosynthetic rates in nutrient-deficient soils El-Sharkawy. ${ }^{3}$ These characteristics make it suitable for both small and large-scale cultivation with moderate agricultural inputs. However, sustainable cassava production especially in the face of both increasing reliance on fertilizers and recurring environmental issues associated with their long-term use requires exploring alternative approaches that could help meet the food demands of rapidly expanding populations. The use of organic residues in combination with mineral fertilizers is a widely accepted soil fertility management strategy Edmeades, ${ }^{4}$ Diacono et al..$^{5}$ Šimon et al. ${ }^{6}$ This practice is known to improve biological, chemical and physical properties of agricultural soils 
as opposed to the sole application of inorganic fertilizers Kotschi; ${ }^{7}$ Mulvaney et al. ${ }^{8}$ In fact, consistently lower cassava yields have been recorded under sole inorganic fertilizer application compared to when organic manures are combined with inorganic fertilizers (Ayoola and Adeniyan 2006; Ojeniyi et al. ${ }^{9}$ For example, Ojeniyi et al. ${ }^{9}$ reported that combination of $2.5 \mathrm{t} / \mathrm{ha}$ of poultry manure and one quarter less the recommended amount of inorganic fertilizer (NPK $15-15-15 ; 600 \mathrm{~kg} / \mathrm{ha}$ ) led to twofold increase in yield of cassava compared to sole application of the same inorganic fertilizer at 600 $\mathrm{kg} / \mathrm{ha}$ after 6 months of cultivation. Similarly, Islami et al., (2011) reported that the application of chemical fertilizers in monoculture cassava was inadequate to maintain sustained yields; but combining farm yield manure (FYM) with chemical fertilizers increased soil fertility and cassava production. Arbuscular Mycorrhizal Fungi (AMF) also referred to as Vesicular Arbuscular Mycorrhiza (VAM) are widespread in terrestrial ecosystems and form mutually beneficial associations with nearly $80 \%$ of higher plants Smith et al. ${ }^{10}$ They are characterized by their intercellular and intracellular growth forms in plant roots, which are referred to as vesicles and arbuscules Böhm et al. ${ }^{11}$ Mycorrhizal symbioses are known to mitigate the problem of efficient uptake of immobile nutrients by plants Bolan; ${ }^{12}$ Smith et al. ${ }^{13}$ Previous studies have shown that AMF are associated with salt Carretero et al. ${ }^{14}$ And drought tolerance Qiangsheng et al. ${ }^{15}$ RuizLozano et al. ${ }^{16}$ and cassava is known to thrive under these conditions. The potentials of AMF in sustainable crop production have been demonstrated by many scholars Li et al. ${ }^{17}$ Céli et al., 2016; Tchabi et al. ${ }^{18}$ However its applicability has not been fully addressed.

Cassava tolerates low soil nutrient levels. However, this crop needs substantial fertilization to attain high yields like many other food crops Howeler. ${ }^{19}$ Perhaps, cassava would not have been a successful crop under both fertilized and un-fertilized conditions without its dependency on mycorrhizal fungi for nutrient uptake, especially phosphate uptake Habte et al. ${ }^{20}$ These authors reported cassava mycorrhizal dependency (that is, the change in cassava growth due to arbuscular mycorrhizal colonization) of $60 \%$ in contrast to the much lower values (44-46\%) obtained in many crop species Tawaraya. ${ }^{21}$ Recently, Burns et al. (2012) showed that mycorrhizal dependency of cassava could reach up to $93 \%$. This dependency is indicative of the wide variety of AMF associated with cassava roots as Glomus, Gigaspora and Acaulospora which are the most commonly reported genera Straker et al. ${ }^{22}$ Bi Voko et al., ${ }^{23}$ dos Santos Heberle et al., ${ }^{24}$ Begoude et al. ${ }^{25}$ Several species in the genus Glomus including G. manihotis Sieverding et al. ${ }^{26}$ G. fasciculatum, G. clarum et al. ${ }^{27}$ $G$. deserticola Okon et al., ${ }^{28}$ G. intraradices Carretero et al.; ${ }^{29} G$. aggregatum $^{20}$ and G. etunicatum Salami et al., ${ }^{30}$ have been tested in field studies with or without fertilization. Obviously, cassava's poor root system architecture, a factor that is crucial in nutrient uptake and subsequent productivity in many crops Smith et al. ${ }^{10}$

Is a major cause of its dependency on mycorrhiza for nutrient acquisition. The beneficial effects of AMF association with cassava have been the subject of several studies. Howeler et al., ${ }^{31}$ Reported a significant increase in growth and dry matter content of mycorrhizainoculated cassava cuttings compared to non-inoculated plants, which were P-deficient even at high P soil levels, Sieverding et al., ${ }^{26}$ Found that N-P-K concentration ratios in cassava shoots and roots were more balanced in mycorrhiza-inoculated plants than in non-inoculated plants. In the same vein, Ceballos et al. (2013) showed that not only $20 \%$ increase in cassava yield was obtained due to addition of Rhizophagus irregulars but also a $50 \%$ reduction in phosphate fertilizer. Other beneficial effects have been reported including enhanced plantlet survival, shoot, root and tuber formation Azcón-Aguilar et al.; ${ }^{32}$ salt damage alleviation Carretero et al. ${ }^{29}$ and resistance to transplant stress Carretero et al. ${ }^{14}$ Given the growing body of evidence on the beneficial effects of AMF on cassava, the present study tested effects of AMF and its combination with organic and inorganic fertilizers to improve cassava productivity in a sustainable manner. Therefore this study hypothesized that soil inoculated with Glomus deserticola will enhance cassava growth.

\section{Materials and methods}

\section{Study area}

This study was carried out at the Botany Research Farm, Department of Botany, University of Ibadan ( $7^{\circ} 26.44^{\prime}$ N, $3^{0} 53.76^{\prime}$ E) between 18 July 2016 and 18 January 2017 . The University has mean annual rainfall and temperature of $1316 \mathrm{~mm}$ and $27.6^{\circ} \mathrm{C}$ respectively.

\section{Soil and cow dung analyses}

Soil samples were randomly taken from $0-20 \mathrm{~cm}$ depth before planting, bulked, air-dried and sieved using $2 \mathrm{~mm}$ sieve for analysis. The particle size analysis was done by pipette method Gee et al. ${ }^{33}$ Soil $\mathrm{pH}$ in water was determined using soil: water ratio of $1: 2$ with a glass electrode $\mathrm{pH}$ meter. Organic carbon was determined using Walkey and Black method (Nelson and Sommers, 1996). Total nitrogen (N) in the soil was determined by Kjedahl digestion Bremner. ${ }^{34}$ Exchangeable bases in the samples were extracted in $1 \mathrm{M} \mathrm{NH}_{4} \mathrm{OAC}$ at $\mathrm{pH}$ 7.0. Calcium $(\mathrm{Ca})$ and magnesium $(\mathrm{Mg})$ in the extract were read by atomic absorption spectrophotometer (AAS). Sodium (Na) and potassium (K) were analyzed by flame photometry. Available phosphorus (P) was determined by Bray-1 extraction and determined colourimetrically by the molybdenum blue procedure Bray et al. ${ }^{35}$

Cow dung samples were air-dried and ground to powder and analysed with wet digestion method using 5:1:1 $\mathrm{ml}$ of $\mathrm{HNO}_{3}$ : $\mathrm{H}_{2} \mathrm{SO}_{4}: \mathrm{HClO}_{4}$ acid. Total $\mathrm{N}$ was determined by micro-Kjeldahl method (Jackson, 1962). For P, K, Ca and Mg, samples $(0.5 \mathrm{~g}$ ) were ashed, dissolved in $10 \%$ hydrogen chloride $(\mathrm{HCl})$ and diluted to $50 \mathrm{ml}$. Phosphorous was determined using vandal molybdate colorimetric. Calcium and Magnesium were determined by EDTA titration while $\mathrm{Na}$ and $\mathrm{K}$ by flame photometry. The physico-chemical properties of both soil and cow dung used were analyzed at the Department of Agronomy, University of Ibadan.

\section{Experimental design and treatment application}

The experiment involved seven treatments: AMF (Glomus deserticola) 20, 30, 40 and 50g), NPK 15:15:15 40, 60, 80 and 100g; cow dung 200, 300, 400 and 500g; AMF and NPK 20+40, 30+60, $40+80$ and $50+100 \mathrm{~g}$, AMF and cow dung $20+200,30+300,40+400$, $50+500 \mathrm{~g}$, cow dung and NPK 40+200,60+300, 80+400, 100+500g laid out in a completely randomized design (CRD) with four replicates. The soil was tilled before planting. Cassava stem cuttings (TME 419) of about $15 \mathrm{~cm}$ long were planted horizontally and buried completely at $5 \mathrm{~cm}$ depth in heaps of soil at a planting distance of $1 \mathrm{~m}$ apart in a plot size of $14 \mathrm{~m} \times 14 \mathrm{~m}$. The treatments were applied two weeks after establishment. Application of NPK 15:15:15, cow dung and Glomus deserticola was done using the methods of Ojeniyi et al., ${ }^{9}$ Mathias et al. ${ }^{36}$ and Okon et al. ${ }^{28}$ Respectively, four different levels of each $G$. deserticola $(20 \mathrm{~g}, 30 \mathrm{~g}, 40 \mathrm{~g}$ and 50g); NPK 15:15:15 (40g, 60g, 80g and $100 \mathrm{~g})$ and cow dung $(200 \mathrm{~g}, 300 \mathrm{~g}, 400 \mathrm{~g}$ and $500 \mathrm{~g})$ were applied 
both singly and in combination around the growing stem cuttings.

\section{Data collection}

Data collection commenced two weeks after application of treatments and subsequently forth nightly for five months. The following growth parameters: plant height, stem girth and leaf area were determined forth nightly. Plant height $(\mathrm{cm})$ and leaf area $\left(\mathrm{cm}^{2}\right)$ were determined using a Measuring Tape and Portable Electronic Area Metre Model Li-3000 respectively. Stem girth was measured at $5 \mathrm{~cm}$ above heap level using Mitutoyo Digimatic Electronic calliper (MDEC) Model CD-8"P. The leaf chlorophyll content was determined by using the central leaf from freshly excised leaves of the same age. The excised leaf was weighed, cut into smaller pieces and stored in the dark for $24 \mathrm{hrs}$ in a mixture of $10 \mathrm{ml}$ of $95 \%$ ethanol and $99.5 \%$ acetone $(1: 1 \mathrm{v} / \mathrm{v})$. One millilitre of the concentrated leaf extract was added to a cuvette and adjusted to $5 \mathrm{ml}$ with the ethanol-acetone mixture. The Absorbance of the mixture was measured spectrophotometrically at $652 \mathrm{~nm}$ using a Unico spectrophotometer. This value was taken as reference by resetting the absorbance to zero and measuring that of the diluted leaf extract. Chlorophyll concentration $(\mathrm{ml} / \mathrm{g})$ was estimated using equation (1)

$$
\begin{aligned}
& \text { Chlorophyll Concentration }=\frac{\mathrm{A}_{652}}{34.5} \times \frac{\mathrm{V}}{1000 \mathrm{~W}} \\
& \text { Where A652=Sample OD value (Absorbance at } 652 \mathrm{~nm}) \\
& \text { V=Volume of the sample }(\mathrm{ml}) \\
& \mathrm{W}=\text { Weight of the sample }(\mathrm{g})
\end{aligned}
$$

The yield data was collected six months after planting and these include fresh and dry weight of tubers. The plants were harvested and separated into roots and stems. Dry weight was determined after oven drying at $100^{\circ} \mathrm{C}$ for 48 hours using Ohaus Sensitive Electronic Digital Weighing Balance Model SPX2202.

\section{Data analysis}

Data were analysed using Minitab 16 Statistical Software (2010). A one way Analysis of Variance (ANOVA) was carried out to test the effects of the treatments on cassava growth and development. Means were separated using Tukey's HSD test $(\mathrm{p} \leq 0.05)$.

\section{Results}

Table 1 shows the values of the soil physicochemical properties of the experimental site and the nutrient composition of cow dung used in the study. The soil was silt loamy and slightly acidic. Total nitrogen and Organic carbon were higher than $0.11 \%$ and $2 \%$ respectively, which are critical values for Nigerian soils Adepetu. ${ }^{37}$ The soil was poor in Phosphorus (Available $\mathrm{P}<10 \mathrm{mg} / \mathrm{Kg}$ ) while potassium was detected in adequate amounts (Exchangeable $\mathrm{K}>0.2 \mathrm{C}$ mol (+)/Kg. Effective CEC (sum of equivalent charge concentrations of cations $\left(\mathrm{Ca}^{2+}, \mathrm{Mg}^{2+}, \mathrm{K}^{+} \mathrm{Na}^{+}\right.$and $\left.\mathrm{Al}^{3+}\right)$ was low (i.e between 5-15C mol $\left.(+) \mathrm{Kg}^{-1}\right)$,Cassava plant height $(\mathrm{cm})$ The study showed significant differences in plant height from 8 weeks after establishment (WAE). Between 8 to $12 \mathrm{WAE}$, three main effects were distinguished. AMF and NPK combination ( $30 \mathrm{~g}$ of AMF and $60 \mathrm{~g}$ of NPK) significantly enhanced plant height $(133 \pm 8,145 \pm 10$ and $149 \pm 13 \mathrm{~cm}$ respectively) On the other hand, sole application of cow dung did not produce plants with appreciable increase in height $(57 \pm 9,71 \pm 8$ and $70 \pm 14 \mathrm{~cm}$ respectively). Other treatments produced plant heights similar to the control. On the other hand, from 14WAE to 20WAE, the plants showed decreasing performance in height in the order AMFNPKL2 $(30+60) \mathrm{g}>\mathrm{NPK} 100 \mathrm{~g}$ and CDNPKL4 $(500+100) \mathrm{g}>$ control $>\mathrm{CD}(400$ g) $>$ CD (200g) Table 2.

\begin{tabular}{|c|c|c|}
\hline Parameter & Soil & Cow dung \\
\hline $\mathrm{pH}$ & 5.86 & - \\
\hline Nitrate & $4.66 \mathrm{~g} / \mathrm{Kg}$ & $1.81 \%$ \\
\hline Organic carbon & $54.75 \mathrm{~g} / \mathrm{Kg}(\mathrm{H})$ & - \\
\hline Phosphate & - & $0.53 \%$ \\
\hline Average phosphorus & $6.52 \mathrm{mg} / \mathrm{Kg}(\mathrm{L})$ & - \\
\hline $\begin{array}{l}\text { Exchangeable } \\
\text { aluminium }\end{array}$ & $0.5 \mathrm{C} \mathrm{mol}(+) / \mathrm{Kg}$ & - \\
\hline Calcium & $8.86 \mathrm{C} \mathrm{mol}(+) / \mathrm{Kg}$ & $0.28 \%$ \\
\hline Magnesium & $0.69 \mathrm{C} \mathrm{mol}(+) / \mathrm{Kg}$ & $0.14 \%$ \\
\hline Potassium & $0.55 \mathrm{C} \mathrm{mol}(+) / \mathrm{Kg}$ & $1.04 \%$ \\
\hline Sodium & $0.18 \mathrm{C} \mathrm{mol}(+) / \mathrm{Kg}$ & $0.22 \%$ \\
\hline Manganese & $17.9 \mathrm{mg}(+) / \mathrm{Kg}$ & $2160 \mathrm{mg}(+) / \mathrm{Kg}$ \\
\hline Iron & $224 \mathrm{mg}(+) / \mathrm{Kg}$ & 17.5 mg(+)/Kg \\
\hline Copper & $1.34 \mathrm{mg}(+) / \mathrm{Kg}$ & $15.05 \mathrm{mg}(+) / \mathrm{Kg}$ \\
\hline Zinc & $7.83 \mathrm{mg}(+) / \mathrm{Kg}$ & $1625 \mathrm{mg}(+) / \mathrm{Kg}$ \\
\hline Silt & $1560 \mathrm{~g} / \mathrm{Kg}$ & - \\
\hline Clay & $116 g / K g$ & - \\
\hline Sand & $728 \mathrm{~g} / \mathrm{Kg}$ & - \\
\hline
\end{tabular}

Table I Physico-chemical characteristics of the soil at the experimental site, Botany Research Farm, University of Ibadan and chemical composition of cow dung

\section{Cassava leaf area $\left(\mathrm{cm}^{2}\right)$}

There was a general increase in leaf area from 8-12WAE. However, from 14-20WAE, the leaf area showed general decrease in size. On the other hand, there were significant differences at 8 and 10WAE among treatments for plants with AMF and NPK combination $(30+60 \mathrm{~g})$ having the widest leaf areas while plants treated with cow dung $(400 \mathrm{~g})$ showed the least leaf area. Although at 12WAE and 1620WAE, no significant differences among treatments were observed. It is important to note that the interaction of AMF with any combination of treatment produced plants with big leaf areas (Table 3 ).

\section{Cassava stems girth $(\mathrm{mm})$}

Significant differences in plant stem girth were observed from 10 20 WAE. The combination of AMF and inorganic fertilizer (30g of AMF and $60 \mathrm{~g}$ of NPK) produced plants with the largest stem girth while plants treated with cow dung $(200 \mathrm{~g})$ produced the least girth. Stem girth showed similar growth pattern throughout the period of study (Table 4).

\section{Cassava leaf chlorophyll content ( $\mathrm{ml} / \mathrm{g})$}

The effects of different treatments on the leaf chlorophyll content at 10 weeks after planting and establishment of the plants are represented in Figure 1. The mean chlorophyll concentration was highest in the combined application of inorganic fertilizer and G. derserticola $(0.1825 \pm 0.0007 \mathrm{ml} / \mathrm{g})$ and lowest with cow dung in 
combination with $G$. derserticola. Chlorophyll in plants treated with sole NPK was next $(0.15497 \pm 0.000246 \mathrm{ml} / \mathrm{g})$ after the combined application of $G$. deserticola and NPK. Cow dung, G. deserticola and the control treatments had similar effect on leaf chlorophyll content with concentrations of $0.0909 \pm 0.0006,0.0922 \pm 0.0005$ and $0.0925 \pm 0.0004 \mathrm{ml} / \mathrm{g}$ respectively (Figure 1 ).

\section{Yield}

Figure 2 represents the fresh cassava weight obtained from the different treatments. Significant differences were observed among

Table 2 Effects of treatments on cassava plant height $(\mathrm{cm})$

\begin{tabular}{|c|c|c|c|c|c|c|c|}
\hline Treatment & 8WAE & IOWAE & I 2WAE & I 4WAE & I6WAE & I8WAE & 20 WAE \\
\hline Control & $97.33 \pm 19.66^{\mathrm{ab}}$ & $100.10 \pm 16.42^{\mathrm{ab}}$ & $122.42 \pm 5.47^{\mathrm{ab}}$ & $128.98 \pm 2.37^{\mathrm{abc}}$ & $\left.|28.55 \pm| .6\right|^{a b}$ & $|28.62 \pm| .40^{\mathrm{abc}}$ & $13 \mid .15 \pm 2.38^{\mathrm{abc}}$ \\
\hline AMF $20 \mathrm{~g}$ & $85.55 \pm 16.66^{\mathrm{ab}}$ & $94.70 \pm 15.66^{\mathrm{ab}}$ & $100.72 \pm 17.79^{a b}$ & $108.35 \pm 16.38^{\mathrm{abc}}$ & $114.18 \pm 14.93^{\mathrm{ab}}$ & $114.25 \pm 13.64^{\mathrm{abc}}$ & $115.08 \pm 12.35^{\mathrm{abc}}$ \\
\hline AMF $30 \mathrm{~g}$ & $64.98 \pm 5.46^{\mathrm{ab}}$ & $75.08 \pm 6.74^{\mathrm{ab}}$ & $82.70 \pm 6.28 \mathrm{~d}^{\mathrm{ab}}$ & $91.78 \pm 5.26^{\mathrm{abc}}$ & $89.43 \pm 5.76^{\mathrm{ab}}$ & $86.45 \pm 6.35^{\mathrm{abc}}$ & $86.58 \pm 6.2 \mathrm{I}^{\mathrm{abc}}$ \\
\hline AMF 40g & $83.90 \pm 7.18^{\mathrm{ab}}$ & $91.65 \pm 6.08^{\mathrm{ab}}$ & $93.25 \pm 7.13^{\mathrm{ab}}$ & $93.13 \pm 8.05^{\mathrm{abc}}$ & $93.70 \pm 7.18^{\mathrm{ab}}$ & $93.60 \pm\left. 5.7\right|^{a b c}$ & $98.90 \pm 7.02^{\mathrm{abc}}$ \\
\hline AMF $50 \mathrm{~g}$ & $70.10 \pm 16.37^{\mathrm{ab}}$ & $78.38 \pm 14.60^{\mathrm{ab}}$ & $91.13 \pm 11.52^{\mathrm{ab}}$ & $97.50 \pm 9.30^{\mathrm{abc}}$ & $90.78 \pm 6.00^{\mathrm{ab}}$ & $99.30 \pm 9.55^{\mathrm{abc}}$ & $107.22 \pm 11.06^{\mathrm{abc}}$ \\
\hline NPK 40g & $103.12 \pm 10.08^{a b}$ & $113.32 \pm 10.78^{\mathrm{ab}}$ & $130.33 \pm 8.26^{\mathrm{ab}}$ & $132.77 \pm 7.48^{\mathrm{abc}}$ & $134.90 \pm 18.46^{\mathrm{ab}}$ & $136.67 \pm 8.05^{\mathrm{abc}}$ & $137.90 \pm 7.60^{\mathrm{abc}}$ \\
\hline NPK 60g & $113.38 \pm 8.94^{\mathrm{ab}}$ & $90.80 \pm 8.78^{\mathrm{ab}}$ & $121.57 \pm 5.48^{\mathrm{ab}}$ & $120.03 \pm 6.07^{\mathrm{abc}}$ & $122.23 \pm 6.49^{a b}$ & $124.07 \pm 7.34^{\mathrm{abc}}$ & $124.47 \pm 4.30^{\mathrm{abc}}$ \\
\hline NPK 80g & $91.75 \pm 14.52^{\mathrm{ab}}$ & $115.92 \pm 10.7 b$ & $106.78 \pm 14.33^{a b}$ & $108.50 \pm 13.27^{\mathrm{abc}}$ & $99.10 \pm 6.49^{\mathrm{ab}}$ & $99.03 \pm 7.34^{\mathrm{abc}}$ & $115.12 \pm 13.68^{\mathrm{abc}}$ \\
\hline NPK 100g & $\mid 20.85 \pm 1.74^{\mathrm{ab}}$ & $130.52 \pm 20.18^{\mathrm{ab}}$ & $133.22 \pm 22.37^{\mathrm{ab}}$ & $138.45 \pm 20.42^{\mathrm{ab}}$ & $136.95 \pm 19.88^{\mathrm{ab}}$ & $137.70 \pm 17.62^{\mathrm{ab}}$ & $143.05 \pm 20.88^{\mathrm{ab}}$ \\
\hline CD 200g & $57.35 \pm 8.90 b$ & $70.90 \pm 7.55 b$ & $69.75 \pm 14.08 b$ & $65.15 \pm 20.68^{c}$ & $66.60 \pm 19.64 b$ & $65.85 \pm 18.86^{c}$ & $63.70 \pm 18.96^{c}$ \\
\hline CD 400g & $64.58 \pm\left. 6.3\right|^{\mathrm{ab}}$ & $69.98 \pm 7.58 b$ & $71.63 \pm 8.99 b$ & $84.40 \pm 8.40 b^{c}$ & $89.68 \pm 10.38^{\mathrm{ab}}$ & $74.33 \pm 5.79 b^{c}$ & $72.08 \pm 7.43 b^{c}$ \\
\hline CD 500g & $79.60 \pm 6.42^{\mathrm{ab}}$ & $90.25 \pm\left. 9.3\right|^{\mathrm{ab}}$ & $98.45 \pm 14.98^{\mathrm{ab}}$ & $102.15 \pm 16.03^{\mathrm{abc}}$ & $105.68 \pm 16.75^{a b}$ & $107.32 \pm 16.47^{a b c}$ & $110.65 \pm 16.54^{\mathrm{abc}}$ \\
\hline AMFNKPLI $(20+40) g$ & $97.15 \pm|3.2|^{\mathrm{ab}}$ & $97.78 \pm 13.24^{\mathrm{ab}}$ & $108.93 \pm 14.98^{\mathrm{ab}}$ & $1 \mid 4.47 \pm 10.19^{a b c}$ & $116.43 \pm 12.93^{\mathrm{ab}}$ & || $8.97 \pm \mid 3.43^{\mathrm{abc}}$ & $|24| .3 \pm|3.8|^{a b c}$ \\
\hline AMFNKPL2 $(30+60) g$ & $133.22 \pm 7.95 a$ & $145.42 \pm 9.62 \mathrm{a}$ & $|48.92 \pm| 2.8 \mid \mathrm{I}$ & $159.60 \pm 13.09 a$ & $158.87 \pm \mid 2.95 \mathrm{a}$ & $149.70 \pm 8.24 a$ & $165.73 \pm 11.96 a$ \\
\hline AMFNKPL3 $(40+80) g$ & $75.90 \pm 7.87^{\mathrm{ab}}$ & $106.10 \pm 9.38^{\mathrm{ab}}$ & $\mid \mathrm{I} 8.70 \pm 7.8 \mathrm{I}^{\mathrm{ab}}$ & $123.77 \pm 7.44^{\mathrm{abc}}$ & $\mid 21.70 \pm 7.83^{\mathrm{ab}}$ & $120.45 \pm 8.18^{\mathrm{abc}}$ & $121.12 \pm 9.86^{\mathrm{abc}}$ \\
\hline AMFNKPL4 $(50+100) \mathrm{g}$ & $97.38 \pm 12.54^{\mathrm{ab}}$ & $110.45 \pm 13.04^{\mathrm{ab}}$ & $118.72 \pm 12.94^{\mathrm{ab}}$ & $124.45 \pm 1 \mid .46^{\mathrm{abc}}$ & $104.42 \pm 18.63^{\mathrm{ab}}$ & $118.35 \pm 11.84^{\mathrm{abc}}$ & $\left.|10.28 \pm| 3.4\right|^{a b c}$ \\
\hline AMFCDLI $(20+200) g$ & $81.73 \pm\left. 15.1\right|^{\mathrm{ab}}$ & $91.15 \pm 13.24^{\mathrm{ab}}$ & $99.33 \pm 13.77^{\mathrm{ab}}$ & $102.55 \pm 13.54^{\mathrm{abc}}$ & $109.95 \pm 14.60^{\mathrm{ab}}$ & $|1| .62 \pm \mid 4.23^{\mathrm{abc}}$ & $|\mathrm{I} 2.72 \pm| 4.6 \mathrm{I}^{\mathrm{abc}}$ \\
\hline AMFCDL2 $(30+300) \mathrm{g}$ & $91.05 \pm 12.50^{\mathrm{ab}}$ & $102.48 \pm 12.14^{\mathrm{ab}}$ & $108.82 \pm 16.98^{\mathrm{ab}}$ & $107.12 \pm 10.78^{\mathrm{abc}}$ & $95.43 \pm 4.69^{\mathrm{ab}}$ & $100.09 \pm 6.0 I^{\mathrm{abc}}$ & $96.28 \pm 9.37^{\mathrm{abc}}$ \\
\hline AMFCDL3 $(40+400) \mathrm{g}$ & $102.85 \pm|5.1|^{a b}$ & $112.18 \pm 12.87^{a b}$ & $106.48 \pm 17.65^{a b}$ & $115.55 \pm 18.14^{\mathrm{abc}}$ & $118.70 \pm 14.52^{\mathrm{ab}}$ & $122.50 \pm 16.97^{\mathrm{abc}}$ & $130.30 \pm 24.30^{\mathrm{abc}}$ \\
\hline AMFCDL4 $(50+500) \mathrm{g}$ & $66.40 \pm 15.33^{\mathrm{ab}}$ & $76.23 \pm 15.36^{\mathrm{ab}}$ & $89.45 \pm 11.83^{\mathrm{ab}}$ & $91.90 \pm 9.18^{\mathrm{abc}}$ & $90.48 \pm 8.55^{\mathrm{ab}}$ & $89.00 \pm 7.56^{\mathrm{abc}}$ & $92.83 \pm 10.37^{a b c}$ \\
\hline CDNPKL3 $(400+80) g$ & $83.45 \pm 22.92^{\mathrm{ab}}$ & $99.95 \pm 22.53^{\mathrm{ab}}$ & || $10.88 \pm\left. 23.0\right|^{a b}$ & $|26.88 \pm| 4.89^{a b c}$ & $|23.38 \pm 2| .13^{\mathrm{ab}}$ & $|2| .80 \pm\left. 1.0\right|^{a b c}$ & $117.40 \pm 21.66^{\mathrm{abc}}$ \\
\hline CDNPKL4 $(500+100) \mathrm{g}$ & $110.93 \pm 14.44^{\mathrm{ab}}$ & $122.83 \pm 10.99^{\mathrm{ab}}$ & $136.37 \pm 6.00^{\mathrm{ab}}$ & $140.80 \pm 4.32^{\mathrm{ab}}$ & $1 \mid 5.50 \pm 3.88^{\mathrm{ab}}$ & $1 \mid 5.37 \pm 3.86^{\mathrm{abc}}$ & $122.67 \pm 1.68^{\mathrm{abc}}$ \\
\hline
\end{tabular}

Means and standard error of the treatments separated using Tukey's HSD $(p<.05)$. Means with the same letter along the column are not significantly different. AMF, arbuscular mycorrhiza fungi; $C D$, cow dung;WAE, weeks after establishment

Table 3 Effects of treatments on cassava leaf area $\left(\mathrm{cm}^{2}\right)$

\begin{tabular}{|c|c|c|c|c|c|c|c|}
\hline Treatment & 8WAE & IOWAE & I 2WAE & I4WAE & I6WAE & I 8WAE & 20WAE \\
\hline Control & $150.23 \pm 9.99^{a b}$ & $253.65 \pm 62.12^{\mathrm{ab}}$ & $280.65 \pm 67.30^{a}$ & $198.10 \pm 26.53^{\mathrm{ab}}$ & $90.16 \pm 5.67^{a}$ & $75.25 \pm 17.12^{\mathrm{a}}$ & $71.45 \pm 7.58^{\mathrm{a}}$ \\
\hline AMF 20g & $158.90 \pm 10.96^{\mathrm{ab}}$ & $277.86 \pm 39.99^{a b}$ & $146.90 \pm 33.29^{a}$ & $164.88 \pm 20.30^{\mathrm{ab}}$ & $99.39 \pm 5.49^{a}$ & $98.79 \pm 11.30^{\mathrm{a}}$ & $57.99 \pm 11.0 \mathrm{I}^{\mathrm{a}}$ \\
\hline AMF 30g & $118.91 \pm 14.45^{\mathrm{ab}}$ & $|64.6| \pm 29.83^{\mathrm{ab}}$ & $146.96 \pm 25.50^{a}$ & $|35.0| \pm 5.23^{\mathrm{ab}}$ & $84.34 \pm 7.13^{a}$ & $65.27 \pm 13.68^{a}$ & $48.4 I \pm 17.48^{a}$ \\
\hline AMF 40g & $126.06 \pm 6.70^{\mathrm{ab}}$ & $240.28 \pm 15.85^{\mathrm{ab}}$ & $170.95 \pm 25.77^{a}$ & $|19.5| \pm \mid 3.69 b$ & $73.40 \pm 4.60^{\mathrm{a}}$ & $46.49 \pm 6.35^{\mathrm{a}}$ & $74.43 \pm 7.66^{a}$ \\
\hline AMF 50g & $|32.0| \pm \mid 4.00^{\mathrm{ab}}$ & $215.10 \pm 28.02^{\mathrm{ab}}$ & $181.17 \pm 10.42^{\mathrm{a}}$ & $157.34 \pm 8.30^{\mathrm{ab}}$ & $95.58 \pm 9.99^{a}$ & $68.06 \pm 16.36^{a}$ & $74.77 \pm 8.82^{\mathrm{a}}$ \\
\hline
\end{tabular}

Citation: Chukwuka KS, Okechukwu RU, Umukoro BO, et al.Arbuscular mycorrhiza fungi, NPK (I5-I5-I5) and cow dung interaction in sustainable cassava production and food security. Adv Plants Agric Res. 2017;7(4):328-335. DOI: 10.15406/apar.2017.07.00262

the treatments. As in other growth parameters, the highest yield was obtained in cassava treated with the mixture of $30 \mathrm{~g}$ of $G$. deserticola and $60 \mathrm{~g}$ of NPK $(0.87 \pm 0.07 \mathrm{Kg})$ while the lowest yield was observed in all the cow dung treatments. The control $(0.23 \pm 0.05 \mathrm{Kg})$ was comparable to all other treatments. The figure also show that dry weight of the tubers follow the same pattern as the fresh weight with the combined AMF-NPK (30 g+60 g) producing the highest dry weight $(0.17 \pm 0.01 \mathrm{Kg})$ while cow dung $(300 \mathrm{~g})$ alone produced the lowest tuber dry weight $(0.008 \pm 0.006 \mathrm{Kg})$. 
Table Continued.

\begin{tabular}{|c|c|c|c|c|c|c|c|}
\hline Treatment & 8WAE & IOWAE & I2WAE & I4WAE & I6WAE & I8WAE & 20WAE \\
\hline NPK 40g & $152.34 \pm 23.37^{\mathrm{ab}}$ & $323.27 \pm 41.12^{\mathrm{ab}}$ & $283.57 \pm 56.37^{a}$ & $178.63 \pm 54.82^{\mathrm{ab}}$ & $109.57 \pm 5.26^{\mathrm{a}}$ & $80.21 \pm|8.3|^{\mathrm{a}}$ & $85.42 \pm 3.12^{\mathrm{a}}$ \\
\hline NPK 60g & $174.94 \pm 26.22^{\mathrm{ab}}$ & $334.26 \pm 9.38^{\mathrm{ab}}$ & $225.37 \pm 46.52^{\mathrm{a}}$ & $140.24 \pm 35.05^{\mathrm{ab}}$ & $87.65 \pm 4.53^{\mathrm{a}}$ & $95.16 \pm 4.99^{a}$ & $74.1 I \pm 4.50^{\mathrm{a}}$ \\
\hline NPK 80g & $140.68 \pm 6.68^{\mathrm{ab}}$ & $276.43 \pm 6 I .43^{\mathrm{ab}}$ & $245.76 \pm 69.22^{\mathrm{a}}$ & $208.81 \pm 49.33^{\mathrm{ab}}$ & $69.34 \pm 11.65^{a}$ & $45.72 \pm 17.23^{a}$ & $43.62 \pm 12.44^{\mathrm{a}}$ \\
\hline NPK I00g & $180.17 \pm 20.54^{\mathrm{ab}}$ & $341.05 \pm 16.60^{\mathrm{ab}}$ & $307.98 \pm 49.49^{a}$ & $245.45 \pm 40.19^{a b}$ & $85.77 \pm 9.87^{a}$ & $71.65 \pm 23.80^{\mathrm{a}}$ & $72.75 \pm 23.57^{a}$ \\
\hline CD 200g & $120.87 \pm 16.42^{\mathrm{ab}}$ & $166.22 \pm 28.63^{\mathrm{ab}}$ & $108.36 \pm 32.44^{a}$ & $125.38 \pm 24.52^{\mathrm{ab}}$ & $96.77 \pm 15.70^{\mathrm{a}}$ & $68.66 \pm 9.95^{a}$ & $88.69 \pm 15.94^{a}$ \\
\hline CD 300g & $138.59 \pm 28.29 \mathrm{ab}$ & $2|7|. I \pm\left. 50.5\right|^{\mathrm{ab}}$ & $193.75 \pm 26.94^{\mathrm{a}}$ & $|49.18 \pm||.4|^{\mathrm{ab}}$ & $96.88 \pm 5.02^{\mathrm{a}}$ & $85.1 I \pm 14.20^{\mathrm{a}}$ & $48.91 \pm 11.60^{\mathrm{a}}$ \\
\hline CD 400g & $\begin{array}{l}110.31 \pm \\
10.26 b\end{array}$ & $163.34 \pm 27.45 b$ & $127.84 \pm 16.86^{\mathrm{a}}$ & $131.16 \pm 10.94^{\mathrm{ab}}$ & $88.70 \pm 11.65^{\mathrm{a}}$ & $66.74 \pm|4.4|^{\mathrm{a}}$ & $63.44 \pm 19.18^{\mathrm{a}}$ \\
\hline CD 500g & $152.62 \pm 18.10^{\mathrm{ab}}$ & $217.10 \pm 36.35^{\mathrm{ab}}$ & $203.99 \pm 47.20^{\mathrm{a}}$ & $134.81 \pm 19.10^{\mathrm{ab}}$ & $91.51 \pm 13.93^{\mathrm{a}}$ & $65.5 \mathrm{I} \pm 7.07^{\mathrm{a}}$ & $102.22 \pm 32.77^{a}$ \\
\hline AMFNKPLI $(20+40) g$ & $186.48 \pm 13.92^{\mathrm{ab}}$ & $251.47 \pm 39.32^{\mathrm{ab}}$ & $|82.1| \pm\left. 20.2\right|^{a}$ & $169.85 \pm 13.38^{\mathrm{ab}}$ & $71.92 \pm 2.46^{\mathrm{a}}$ & $61.71 \pm 18.25^{\mathrm{a}}$ & $82.02 \pm 2.3 I^{a}$ \\
\hline $\begin{array}{l}\text { AMFNKPL2 }(30+60) \\
g\end{array}$ & $227.50 \pm 30.98 a$ & $405.18 \pm 34.67 a$ & $349.06 \pm 39.69^{a}$ & $34 I .50 \pm 1.45 a$ & $61.8 \pm 39.0^{a}$ & $59.83 \pm 28.26^{a}$ & $61.38 \pm 16.74^{a}$ \\
\hline $\begin{array}{l}\text { AMFNKPL3 }(40+80) \\
g\end{array}$ & $161.87 \pm 12.57^{\mathrm{ab}}$ & $284.93 \pm 38.60^{\mathrm{ab}}$ & $215.56 \pm 33.08^{a}$ & $184.55 \pm 9.70^{\mathrm{ab}}$ & $83.04 \pm 6.23^{a}$ & $61.36 \pm 15.47^{a}$ & $71.59 \pm 11.56^{\mathrm{a}}$ \\
\hline $\begin{array}{l}\text { AMFNKPL4 } \\
(50+100) \mathrm{g}\end{array}$ & $159.06 \pm 1.98^{\mathrm{ab}}$ & $228.64 \pm 53.12^{\mathrm{ab}}$ & $233.76 \pm 39.67 a$ & $212.17 \pm 16.16^{\mathrm{ab}}$ & II $3.57 \pm 6.70^{\mathrm{a}}$ & $49.92 \pm 14.32^{\mathrm{a}}$ & $63.39 \pm 19.99^{a}$ \\
\hline $\begin{array}{l}\text { AMFCDLI }(20+200) \\
g\end{array}$ & $|3| .67 \pm 2 \mid .99^{a b}$ & $212.86 \pm 59.60^{\mathrm{ab}}$ & $|75| .4 \pm\left. 64.1\right|^{a}$ & $188.33 \pm 66.55^{\mathrm{ab}}$ & $104.90 \pm 7.60^{\mathrm{a}}$ & $89.51 \pm 14.12^{\mathrm{a}}$ & $62.28 \pm|2.7|^{a}$ \\
\hline $\begin{array}{l}\text { AMFCDL2 }(30+300) \\
g\end{array}$ & $175.92 \pm 20.74^{\mathrm{ab}}$ & $227.97 \pm 51.84^{\mathrm{ab}}$ & $205.86 \pm 52.05^{a}$ & $137.08 \pm 28.09^{a b}$ & $76.49 \pm 8.02^{\mathrm{a}}$ & $58.97 \pm 6.78^{a}$ & $33.44 \pm 8.43^{\mathrm{a}}$ \\
\hline $\begin{array}{l}\text { AMFCDL3 }(40+400) \\
g\end{array}$ & $172.03 \pm 14.94^{\mathrm{ab}}$ & $282.84 \pm 57.02^{\mathrm{ab}}$ & $237.23 \pm 69.3 \mathrm{I}^{\mathrm{a}}$ & $217.81 \pm 46.00^{\mathrm{ab}}$ & $81.87 \pm 21.65^{a}$ & $95.27 \pm 9.39^{a}$ & $73.75 \pm 13.04^{a}$ \\
\hline $\begin{array}{l}\text { AMFCDL4 }(50+500) \\
g\end{array}$ & $138.90 \pm 20.62^{\mathrm{ab}}$ & $208.48 \pm 37.82^{\mathrm{ab}}$ & $190.26 \pm 33.90^{\mathrm{a}}$ & $132.88 \pm 12.73^{\mathrm{ab}}$ & $79.42 \pm 5.86^{a}$ & $64.16 \pm 10.15^{a}$ & $60.45 \pm 4.87^{a}$ \\
\hline $\begin{array}{l}\text { CDNPKLI }(200+40) \\
g\end{array}$ & $|58.05 \pm 6.9|^{\mathrm{ab}}$ & $308.21 \pm 19.76^{a b}$ & $242.42 \pm 49.45^{\mathrm{a}}$ & $205.83 \pm 62.75^{\mathrm{ab}}$ & $83.4 I \pm \mid 4.06^{a}$ & $70.43 \pm 13.35^{\mathrm{a}}$ & $49.98 \pm 16.07^{a}$ \\
\hline $\begin{array}{l}\text { CDNPKL2 }(300+60) \\
g\end{array}$ & $154.62 \pm 19.24^{\mathrm{ab}}$ & $322.37 \pm 60.63^{a b}$ & $272.03 \pm 59.67^{a}$ & $150.32 \pm 10.35^{\mathrm{ab}}$ & $96.22 \pm 19.7 \mathrm{Ia}$ & $111.28 \pm 24.52^{\mathrm{a}}$ & $101.01 \pm 18.26^{a}$ \\
\hline $\begin{array}{l}\text { CDNPKL3 }(400+80) \\
g\end{array}$ & $|5| .10 \pm 52.28^{\mathrm{ab}}$ & $280.98 \pm 56.74^{\mathrm{ab}}$ & $287.94 \pm 72.75^{\mathrm{a}}$ & $224.69 \pm 31.3 I^{\mathrm{ab}}$ & $104.38 \pm 3.22 \mathrm{a}$ & $110.80 \pm 15.63^{\mathrm{a}}$ & $90.44 \pm 4.04^{a}$ \\
\hline $\begin{array}{l}\text { CDNPKL4 }(500+ \\
100) \mathrm{g}\end{array}$ & $212.93 \pm 25.73^{\mathrm{ab}}$ & $323.77 \pm 64.52^{\mathrm{ab}}$ & $353.08 \pm 44.78 a$ & $247.58 \pm 20.48^{\mathrm{ab}}$ & $85.15 \pm 2.38^{a}$ & $84.71 \pm 16.00^{\mathrm{a}}$ & $82.76 \pm 12.94^{\mathrm{a}}$ \\
\hline
\end{tabular}

Means with the same letter along the column are not significantly different.

AMF, arbuscular mycorrhiza fungi; CD, cow dung;WAE, weeks after establishment

Table 4 Effects of treatments on cassava stemsgirth $(\mathrm{mm})$

\begin{tabular}{|c|c|c|c|c|c|c|}
\hline Treatment & IOWAE & I2WAE & I 4WAE & I6WAE & I 8WAE & 20WAE \\
\hline Control & $|4.83 \pm| .89^{a b}$ & $17.13 \pm 0.62^{\mathrm{ab}}$ & $17.08 \pm 0.44^{\mathrm{ab}}$ & $17.48 \pm 0.60^{\mathrm{ab}}$ & $17.48 \pm 0.64^{\mathrm{ab}}$ & $17.10 \pm 0.54^{\mathrm{ab}}$ \\
\hline AMF 20g & $14.97 \pm 1.53^{\mathrm{ab}}$ & $15.40 \pm 1.75^{\mathrm{ab}}$ & $16.24 \pm 1.75^{\mathrm{ab}}$ & $\left.|5.87 \pm| .6\right|^{\mathrm{ab}}$ & $15.67 \pm 2.17^{\mathrm{ab}}$ & $16.16 \pm 1.29^{\mathrm{ab}}$ \\
\hline AMF $30 \mathrm{~g}$ & $12.55 \pm 0.88 b$ & $12.80 \pm 0.69^{\mathrm{ab}}$ & $12.46 \pm 0.57^{\mathrm{ab}}$ & $13.10 \pm 0.60^{\mathrm{ab}}$ & $12.78 \pm 0.80^{\mathrm{ab}}$ & $12.76 \pm 0.63^{\mathrm{ab}}$ \\
\hline AMF $40 \mathrm{~g}$ & $15.40 \pm 1.06^{\mathrm{ab}}$ & $15.06 \pm 0.97^{\mathrm{ab}}$ & $15.36 \pm 0.57^{\mathrm{ab}}$ & $15.20 \pm 1.05^{\mathrm{ab}}$ & $15.37 \pm 0.38^{\mathrm{ab}}$ & $15.39 \pm 0.60^{\mathrm{ab}}$ \\
\hline AMF $50 \mathrm{~g}$ & $13.12 \pm 1.92^{\mathrm{ab}}$ & $13.81 \pm 1.90^{\mathrm{ab}}$ & $14.85 \pm 1.20^{\mathrm{ab}}$ & $\mid 3.94 \pm 1.08^{\mathrm{ab}}$ & $14.68 \pm 1.28^{\mathrm{ab}}$ & $13.66 \pm 1.04^{\mathrm{ab}}$ \\
\hline NPK 40g & $17.15 \pm 1.22^{\mathrm{ab}}$ & $17.90 \pm 0.98^{\mathrm{ab}}$ & $17.99 \pm 2.15^{\mathrm{ab}}$ & $18.45 \pm 1.23^{\mathrm{ab}}$ & $18.66 \pm 1.16^{\mathrm{ab}}$ & $18.09 \pm 1.50^{\mathrm{ab}}$ \\
\hline NPK 60g & $\mid 7.01 \pm 0.86^{\mathrm{ab}}$ & $17.04 \pm 0.43^{\mathrm{ab}}$ & $17.92 \pm 0.70^{\mathrm{ab}}$ & $18.13 \pm 0.65^{\mathrm{ab}}$ & $18.07 \pm 0.67^{\mathrm{ab}}$ & $17.70 \pm 0.14^{\mathrm{ab}}$ \\
\hline NPK 80g & $\left.|5.53 \pm| .5\right|^{\mathrm{ab}}$ & $|4.78 \pm| .45^{\mathrm{ab}}$ & $15.09 \pm 1.27^{\mathrm{ab}}$ & $13.42 \pm 0.75^{\mathrm{ab}}$ & $14.69 \pm 1.37^{\mathrm{ab}}$ & $14.94 \pm 1.25^{\mathrm{ab}}$ \\
\hline NPK I00g & $17.33 \pm 1.98^{\mathrm{ab}}$ & $17.25 \pm 2.05^{\mathrm{ab}}$ & $17.53 \pm 2.25^{\mathrm{ab}}$ & $18.14 \pm 2.05^{\mathrm{ab}}$ & $17.39 \pm 2.09^{\mathrm{ab}}$ & $|7.5| \pm\left. 2.2\right|^{\mathrm{ab}}$ \\
\hline CD 200g & $11.83 \pm 1.00 \mathrm{~b}$ & $12.32 \pm 1.44 b$ & $10.72 \pm 2.48 b$ & $10.92 \pm 2.26 \mathrm{~b}$ & $10.70 \pm 2.18 b$ & $10.28 \pm 2.19 \mathrm{~b}$ \\
\hline CD 300g & $13.14 \pm\left. 1.2\right|^{\mathrm{ab}}$ & $13.95 \pm 1.02^{\mathrm{ab}}$ & $13.85 \pm 1.26^{\mathrm{ab}}$ & $14.46 \pm 0.68^{\mathrm{ab}}$ & $14.46 \pm 0.57^{\mathrm{ab}}$ & $14.39 \pm 0.68^{\mathrm{ab}}$ \\
\hline
\end{tabular}

Citation: Chukwuka KS, Okechukwu RU, Umukoro BO, et al.Arbuscular mycorrhiza fungi, NPK (I5-I5-I5) and cow dung interaction in sustainable cassava production and food security. Adv Plants Agric Res. 20I7;7(4):328-335. DOI: 10.15406/apar.2017.07.00262 


\begin{tabular}{|c|c|c|c|c|c|c|}
\hline Treatment & IOWAE & I 2WAE & I 4WAE & I6WAE & I 8WAE & 20WAE \\
\hline CD 400g & $13.02 \pm 1.29^{a b}$ & $12.47 \pm 1.28 \mathrm{~b}$ & $13.32 \pm 1.66^{\mathrm{ab}}$ & $13.01 \pm 1.43^{\mathrm{ab}}$ & $12.45 \pm 1.65 b$ & $12.82 \pm 1.53^{\mathrm{ab}}$ \\
\hline CD 500g & $14.99 \pm 0.63^{\mathrm{ab}}$ & $15.0 \mid \pm 0.83^{\mathrm{ab}}$ & $15.84 \pm 1.17^{\mathrm{ab}}$ & $16.16 \pm 1.28^{\mathrm{ab}}$ & $15.93 \pm 1.19 \mathrm{ab}$ & $15.14 \pm 1.64^{\mathrm{ab}}$ \\
\hline AMFNKPLI $(20+40) \mathrm{g}$ & $16.57 \pm 2.38^{\mathrm{ab}}$ & $|6.3| \pm 2.05^{\mathrm{ab}}$ & $|6.5| \pm 2.13^{\mathrm{ab}}$ & $17.40 \pm 1.63^{\mathrm{ab}}$ & $16.65 \pm 1.70^{\mathrm{ab}}$ & $16.42 \pm 1.99^{a b}$ \\
\hline AMFNKPL2 $(30+60) \mathrm{g}$ & $20.72 \pm 1.6 \mathrm{la}$ & $20.14 \pm 1.43 a$ & $20.76 \pm 1.80 \mathrm{a}$ & $21.08 \pm 1.89 a$ & $21.19 \pm 1.56 \mathrm{a}$ & $20.87 \pm 1.93 a$ \\
\hline AMFNKPL3 $(40+80) \mathrm{g}$ & $16.27 \pm 1.70^{\mathrm{ab}}$ & $17.61 \pm 1.00^{\mathrm{ab}}$ & $17.69 \pm 0.87^{\mathrm{ab}}$ & $17.86 \pm 0.88^{\mathrm{ab}}$ & $|7.7| \pm 0.69^{\mathrm{ab}}$ & $17.84 \pm 0.69^{\mathrm{ab}}$ \\
\hline AMFNKPL4 $(50+100) g$ & $15.77 \pm 1.06^{\mathrm{ab}}$ & $16.54 \pm 1.18^{\mathrm{ab}}$ & $16.53 \pm 1.06^{\mathrm{ab}}$ & $|6.7| \pm 0.99^{\mathrm{ab}}$ & $17.06 \pm 1.17^{\mathrm{ab}}$ & $16.16 \pm 1.24^{\mathrm{ab}}$ \\
\hline AMFCDLI $(20+200) g$ & $14.09 \pm 1.68^{\mathrm{ab}}$ & $13.90 \pm 1.77^{\mathrm{ab}}$ & $15.34 \pm 1.55^{\mathrm{ab}}$ & $14.73 \pm 2.12^{\mathrm{ab}}$ & $14.32 \pm 1.63^{\mathrm{ab}}$ & $|4.50 \pm 1.6|^{\mathrm{ab}}$ \\
\hline AMFCDL2 $(30+300) g$ & $15.10 \pm 1.15^{\mathrm{ab}}$ & $15.27 \pm 0.80^{\mathrm{ab}}$ & $14.85 \pm 1.08^{\mathrm{ab}}$ & $14.63 \pm 0.79^{\mathrm{ab}}$ & $15.75 \pm 0.72^{\mathrm{ab}}$ & $14.57 \pm 1.22^{\mathrm{ab}}$ \\
\hline AMFCDL3 $(40+400) g$ & $16.09 \pm 1.08^{\mathrm{ab}}$ & $15.62 \pm 1.94^{\mathrm{ab}}$ & $15.80 \pm 1.82^{2 \mathrm{~b}}$ & $16.54 \pm 1.86^{\mathrm{ab}}$ & $16.38 \pm 1.73^{\mathrm{ab}}$ & $|5.78 \pm 2.3|^{\mathrm{ab}}$ \\
\hline AMFCDL4 $(50+500) \mathrm{g}$ & $13.67 \pm 1.64^{\mathrm{ab}}$ & $14.11 \pm 1.20^{\mathrm{ab}}$ & $13.64 \pm 0.69^{\mathrm{ab}}$ & $13.79 \pm 0.68^{\mathrm{ab}}$ & $13.82 \pm 0.78^{\mathrm{ab}}$ & $13.86 \pm 0.94^{\mathrm{ab}}$ \\
\hline CDNPKLI $(200+40) \mathrm{g}$ & $16.54 \pm 0.90^{\mathrm{ab}}$ & $16.91 \pm 1.46^{\mathrm{ab}}$ & $17.17 \pm 1.74^{\mathrm{ab}}$ & $|7.4| \pm 1.86^{\mathrm{ab}}$ & $17.52 \pm 1.97^{\mathrm{ab}}$ & $17.58 \pm 2.15^{\mathrm{ab}}$ \\
\hline CDNPKL2 $(300+60) \mathrm{g}$ & $16.65 \pm 1.22^{\mathrm{ab}}$ & $17.40 \pm 1.23^{\mathrm{ab}}$ & $|6.69 \pm 0.8|^{\mathrm{ab}}$ & $16.74 \pm 0.85^{\mathrm{ab}}$ & $17.75 \pm 2.46^{\mathrm{ab}}$ & $|6.7| \pm 0.97^{\mathrm{ab}}$ \\
\hline CDNPKL3 $(400+80) g$ & $16.27 \pm 2.60^{\mathrm{ab}}$ & $16.68 \pm 2.44^{\mathrm{ab}}$ & $15.88 \pm 2.43^{\mathrm{ab}}$ & $17.38 \pm 2.15^{\mathrm{ab}}$ & $16.97 \pm 2.09^{\mathrm{ab}}$ & $17.34 \pm 2.15^{\mathrm{ab}}$ \\
\hline CDNPKL4 $(500+100) g$ & $|8.32 \pm 1.2|^{\mathrm{ab}}$ & $18.47 \pm 0.52^{\mathrm{ab}}$ & $19.29 \pm 0.65^{\mathrm{a}}$ & $14.09 \pm\left. 2.1\right|^{\mathrm{ab}}$ & $16.76 \pm 2.94^{\mathrm{ab}}$ & $15.99 \pm 2.83^{\mathrm{ab}}$ \\
\hline
\end{tabular}

Means and standard error of the treatments separated using Tukey's HSD $(p<.05)$. Means with the same letter along the column are not significantly different. AMF, arbuscular mycorrhiza fungi; $C D$, cow dung;WAE, weeks after establishment

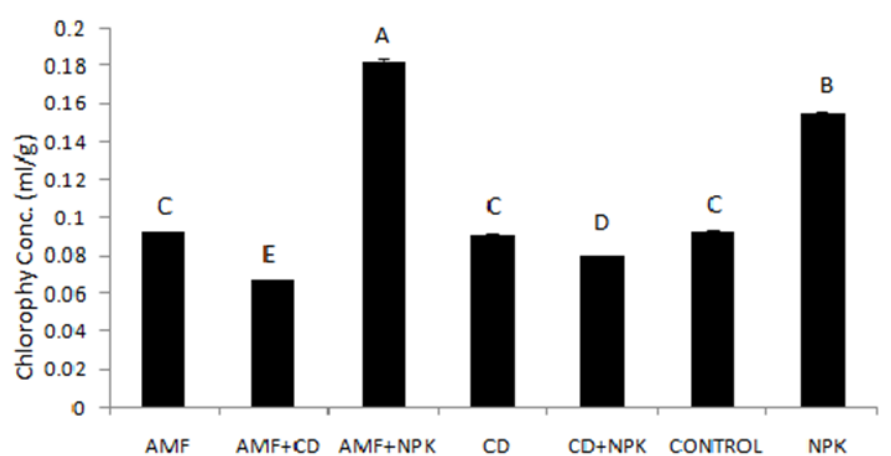

Figure I Effects of treatment on the cassava leaf chlorophyll content $(\mathrm{ml} / \mathrm{g})$. AMF, Arbuscular mycorrhiza fungi; CD, cow dung; NPK, inorganic nitrogen, phosphorus, potassium fertilizer (I5:15:15)

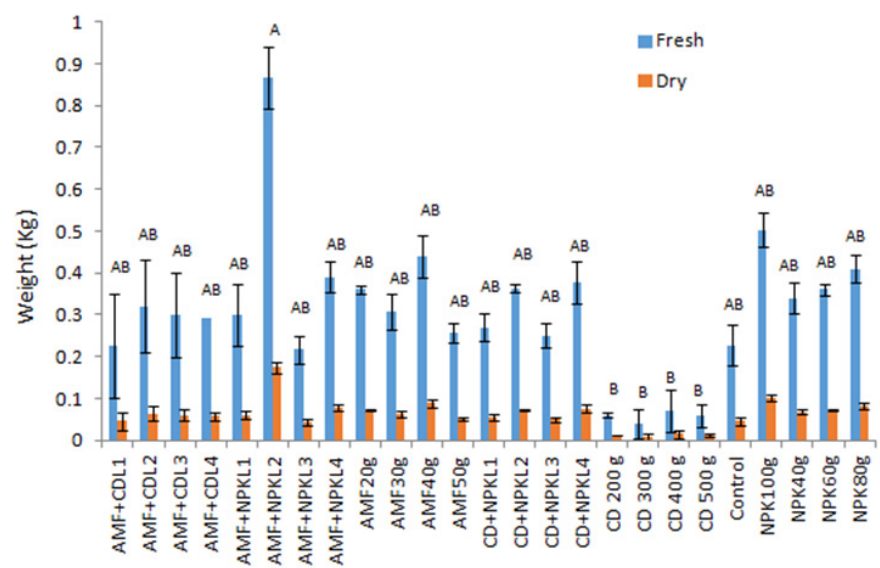

Figure 2 Effects of treatments on cassava yield.

AMF, Arbuscular mycorrhiza fungi; CD, cow dung; NPK, inorganic nitrogen, phosphorus, potassium fertilizer (I5:I5:I5)

\section{Discussion}

Several researchers have demonstrated the beneficial effects of mycorrhizal inoculation on growth and yield of cassava Douds et al. ${ }^{38}$ Carretero et al.; ${ }^{14}$ Séry et al. ${ }^{39}$ However, the combination of AMF and organic/inorganic fertilizers has not been adequately investigated. The results of this study suggest that G. deserticola inoculation in combination with inorganic fertilizer application at the rate of $(30 \mathrm{~g}$ and $60 \mathrm{~g}$ respectively) has beneficial effects on all the growth parameters studied. In this study, leaf chlorophyll content was a good indicator of growth and this was reflected in the yield of the cassava plants studied. Ekanayake et al., ${ }^{24}$ reported that soil inoculated with $10 \mathrm{~g}$ of Glomus clarum and G. mosseae enhanced chlorophyll production in young cassava plants, with the former species supporting more chlorophyll synthesis than the later. Howeler et al., ${ }^{31}$ reported an increase in cassava growth under different combinations of $\mathrm{P}$ input with AMF as opposed to the sole application of AMF. Most of the soils supporting cassava cultivation in the south-western region of the Nigeria are $\mathrm{P}$ deficient thereby underscoring the need for strategies for sustainable soil fertility techniques Salami et al. ${ }^{40}$ The physicochemical properties of the experimental soil do not differ considerably from those reported by these authors.

Arbuscular mycorrhiza fungi are crucial component of the soil ecosystem that enhances nutrient uptake and absorption Bolan, ${ }^{12}$ The increase in growth attributes recorded in this study could be as a result of these processes. Similarly, Sieverding et al., ${ }^{26}$ opined that mycorrhiza inoculation enhanced nutrient uptake in the shoot of cassava compared to non-inoculated plants. In this study, $G$. deserticola inoculation enhanced the growth and yield of cassava therefore supports reduced inorganic fertilizers inputs. This result compares favourably with previous studies on the effects of mycorrhizal inoculations on cassava growth. Ceballos et al. (2013) showed that Rhizophagus irregularis inoculation produced $20 \%$ increase in cassava yield and $50 \%$ reduction in phosphate fertilizer. Similarly Sridevi et al. ${ }^{41}$ studying the response of cassava to Glomus fasciculatum inoculation at increased 
NPK levels reported that yield attributes, like number of tubers, tuber yield were optimal under increased NPK and AMF application. In the present study, inoculation of $G$. deserticola enhanced the performance and yield the cassava plants studied. ${ }^{42-44}$

\section{Conclusion}

The cassava variety TME 419 used in this study responded positively to $G$. deserticola inoculation in combination with inorganic fertilization. This finding indicates the potentials of arbuscular mycorrhiza fungi as a biological agent for sustainable agriculture.

\section{Acknowledgements}

None.

\section{Conflict of interest}

The author declares no conflict of interest.

\section{References}

1. Montagnac JA, Davis CR, Tanumihardjo SA. Nutritional value of cassava for use as a staple food and recent advances for improvement comprehensive reviews. In Food Science And Food Safety. 2009;8(3):181194.

2. FAOSTAT. Statistical Database of the Food and Agriculture Organization of the United Nations. USA; 2016.

3. El-Sharkawy MA. International research on cassava photosynthesis, productivity, eco-physiology, and responses to environmental stresses in the tropics. Photosynthetica. 2006;44(4):481-512.

4. Edmeades DC. The long-term effects of manures and fertilisers on soil productivity and quality: a review. Nutrient Cycling in Agroecosystems. 2003;66(2):165-180.

5. Diacono M, Montemurro F. Long-term effects of organic amendments on soil fertility A review. Agron Sustain Dev. 2010;30(2):401-422.

6. Šimon, T Czakó A. Influence of long-term application of organic and inorganic fertilizers on soil properties. Plant Soil Environ. 2014;60(7):314-319.

7. Kotschi J. A Soiled Reputation Adverse impacts of mineral fertilizers in tropical agriculture AGRECOL-Association for Agriculture and Ecology. Heinrich Böll Stiftung, Germany: WWF; 2013. p. 1-57.

8. Mulvaney RL, Khan SA, Ellsworth TR. Synthetic nitrogen fertilizers deplete soil nitrogen: a global dilemma for sustainable cereal production. $J$ Environ Qual. 2009;38(6):2295-2314.

9. Ojeniyi SO, Adejoro SA, Ikotun O, et al. Soil and plant nutrient composition, growth and yield of cassava as influenced by integrated application of NPK fertilizer and poultry manure. New York Science Journal. 2012;5(9):62-68.

10. Smith S, De Smet I. Root system architecture:insights from Arabidopsis and cereal crops. Phil Trans R Soc B. 2012;367:1441-1452.

11. Böhm J, Hock B. Mycorrhizae: Endomycorrhizae. Progress in Botany. 1997;58:555-594.

12. Bolan NS. A critical review on the role of mycorrhizal fungi in the uptake of Phosphorus by plants. Plant and Soil. 1991;134:(2):189-207.

13. Smith SE, Read DJ. The mycorrhizal symbiosis San Diego, USA: Academic Press; 2008.

14. Carretero CL, Cantos M, Garcia JL, et al. Growth response of micro propagated cassava clones as affected by Glomus intraradices colonization. Journal of Plant Nutrition. 2009;32(2):261-273.
15. Qiangsheng W, Renxue X, Zhengjia H. Effect of arbuscular mycorrhiza on the drought tolerance of poncirus trifoliata seedlings. Frontiers of Forestry in China. 2006;1(1):100-104.

16. Ruiz-Lozano JM, Azcon R, Gomez M. Effects of arbuscular-mycorrhizal glomus species on drought tolerance: physiological and nutritional plant responses. Appl Environ Microbiol. 1995;61(2):456-460.

17. Li H, Ye ZH, Chan WF, et al. Can arbuscular mycorrhizal fungi improve grain yield, as uptake and tolerance of rice grown under aerobic conditions? Environ Pollut. 2011;159(10):2537-2545.

18. Tchabi A, Hountondji FCC, Ogunsola B, et al. The influence of arbuscular mycorrhizal fungi inoculation on micro-propagated hybrid yam (Dioscorea spp ) growth and root knot nematode (Meloidogyne spp) Suppression. Int J Curr Microbiol App Sci. 2016;5(10):267-281.

19. Howeler RH. Long-term effect of cassava cultivation on soil productivity. Field Crops Research. 1991;26:1-18.

20. Habte M, Byappanahalli MN. Dependency of cassava (Manihot esculanta Crantz) on vesicular-arbuscular mycorrhizal fungi Mycorrhiza. 1994;4:241-245

21. Tawaraya K. Arbuscular mycorrhizal dependency of different plant species and cultivars. Soil Sci Plant Nutl. 2003;49(5):655-668.

22. Straker CJ, Hilditch AJ, Rey MEC. Arbuscular mycorrhizal fungi associated with cassava (Manihot esculenta Crantz) in South Africa. South African Journal of Botany. 2010;76(1):102-111.

23. Bi Voko DRR, Nandjui J, Sery JMD, et al. Abundance and diversity of Arbuscular mycorrhizal fungal (AMF) communities associated with cassava (Manihot esculenta Crantz) rhizosphere in Abengourou, East Côte d'Ivoire. Journal of Ecology and the Natural Environment. 2013;5(11):360-370.

24. dos Santos Heberle E, de Armas RD, Heberle DA, et al. Occurrence and Structure of Arbuscular Mycorrhizal Fungal Communities in Cassava after Cultivation of Cover Crops as Observed by the "PCR-DGGE". Technique R Bras Ci Solo. 2015;39(5):1292-1301.

25. Begoude DAB, Sarr PS, Mpon TLY, et al. Composition of arbuscular mycorrhizal fungi associated with cassava (Manihot esculenta Crantz) cultivars as influenced by chemical fertilization and tillage in Cameroon. J Appl Biosci. 2016;98:9270-9283.

26. Sieverding E, Toro ST. Effect of mixing VAM inoculum with fertilizers on cassava nutrition and VAM fungal association. Agriculture Ecosystems and Environment Netherlands. 1989;29(1-4):397-401.

27. Ekanayake IJ, Oyetunji OJ, Osonubi O, et al. The effects of arbuscular mycorrhizal fungi and water stress on leaf chlorophyll production of cassava (Manihot esculenta Crantz), Food, Agriculture \& Environment. 2004;2(2):190-196.

28. Okon IE, Solomon MG, Osonubi O. The effects of arbuscular mycorrhizal fungal inoculation and mulch of contrasting chemical composition on the yield of cassava under humid tropical conditions. Scientific WorldJournal. 2010;10:505-511.

29. Carretero CL, Cantos M, García JL. et al. Arbuscular-mycorrhizal contributes to alleviation of salt damage in cassava clones. Journal of Plant Nutrition. 2008;31(5):959-971.

30. Salami AO, Odebode AC, Osonubi O. The use of Arbuscular Mycorrhiza (AM) as a source of yield increase in sustainable alley cropping system. Archives of Agronomy and Soil Science August. 2005;51(4):385-390.

31. Howeler RH, Edwards DG, Asher CJ. The effect of soil sterilization and mycorrhizal inoculation on the growth, nutrient uptake and critical $P$ concentration of cassava. Intern Symp Trop Root and Tuber Crops, Manila; 1979. p. 519-537. 
32. Azcón-Aguilar C, Cantos M, Troncoso A, et al. Beneficial effect of arbuscular mycorrhizas on acclimatization of micropropagated cassava plantlets. Scientia Horticulturae. 1997;72:63-71.

33. Gee GW, Bauders A. Particle size analysis. In: Khite A editor. Methods of soil analysis, Part 1. 2nd ed. Agronomy Monograph 9, USA; 1986.

34. Bremner JM. N-total In: Sparks DL editor. Methods of soil analysis. Part 3, Soil Science Society of America, USA: SSSA and ASA; 1996. p. 1085-1121.

35. Bray RH, Kurtz LT. Determination of total, organic and available form of phosphorus in soils. Soil Science Society of American Journal. 1945;59(1):39-46.

36. Mathias L, Kabambe VH. Potential to increase cassava yields through cattle manure and fertilizer application: Results from Bunda College. Central Malawi African Journal of Plant Science. 2015;9(5):228-234.

37. Adepetu JA. Interpretation of soil test data: Simple Soil, water and plant testing techniques for soil resource management. In: Depetu JA, et al. editors. Proceedings of a training course, Nigeria; 1996. p 1-158.

38. Douds DD, Nagahashi G, Pfeffer PE, et al. On-farm production and utilization of arbuscular mycorrhizal fungus inoculum. Canadian Journal of Plant Sciences. 2005;85:15-21.
39. Séry DJM, Kouadjo ZGC, Voko BRR Zézé A. Selecting native arbuscular mycorrhizal fungi to promote cassava growth and increase yield under field conditions. Frontiers in Microbiology. 2016;7:2063.

40. Salami BT, Sangoyomi TE. Soil fertility status of cassava fields in South Western Nigeria. American Journal of Experimental Agriculture. 2013;3(1):152-164.

41. Sridevi S, Ramakrishnan K. Effect of inoculation with NPK fertilizer and arbuscular mycorrhizal fungi on growth and yield of cassava. International Journal of Development Research. 2013;3(9):46-50.

42. Anna EB, Roslyn MG, Anabela MZ, et al. Variations in the chemical composition of cassava (Manihot esculenta Crantz) leaves and roots as affected by genotypic and environmental variation. J Agric Food Chem. 2012;60(19):4946-4956.

43. Cely MVT, de Oliveira AG, de Freitas VF, et al. Inoculant of Arbuscular Mycorrhizal Fungi (Rhizophagus clarus) increase yield of soybean and cotton under field conditions. Front Microbiol. 2016;7:1-720.

44. Nelson DW, Sommers LE. Total carbon, organic carbon and organic matter In: Spark DL editor. Methods of soil analysis. Part 3 Chemical Methods, SSSA Book Series No 5 ASA \& SSSA, USA; 1996. p. 961-1010. 